\title{
The Role of Magnetic Buoyancy in a Babcock-Leighton Type Solar Dynamo
}

\author{
Dibyendu Nandy* \& Arnab Rai Choudhuri, Department of Physics, Indian \\ Institute of Science, Bangalore 560012. \\ *e-mail:dandy@physics.iisc.ernet.in
}

\begin{abstract}
We study the effects of incorporating magnetic buoyancy in a model of the solar dynamo-which draws inspiration from the BabcockLeighton idea of surface processes generating the poloidal field. We present our main results here.
\end{abstract}

Key words. MHD—Sun: interior, magnetic fields.

\section{Introduction}

The review by Choudhuri (2000) in these proceedings points out the recent resurgence of interest in Babcock-Leighton (BL) type solar dynamos, in which the poloidal field is assumed to be generated near the solar surface due to the decay of active regions (Choudhuri et al. 1995; Durney 1997; Dikpati \& Charbonneau 1999). Incorporating magnetic buoyancy (MB) in such a model is more tricky than it is in the case of a more traditional dynamo model, where MB could be treated merely as a flux loss mechanism. In a BL type dynamo, MB plays a more central role. It brings the toroidal flux from the bottom of the convection zone (where it is created at the tachocline) to the surface where the active regions thereby formed finally give rise to the poloidal flux. The meridional flow moving poleward and sinking near the poles then brings the poloidal flux back to the bottom of the solar convection zone (SCZ) for the production of toroidal flux. The model of Choudhuri et al. (1995) did not include MB, whereas Durney (1997) and Dikpati \& Charbonneau (1999) treated MB through some simple prescriptions. Here we study the effects of MB by including it in the model of Choudhuri et al. (1995) and then looking for the changes in the results arising out of that. We present the details of our model in the next section.

\section{Model}

We solve the following equations within the northern quadrant of the SCZ (i.e. within $\left.R_{b}=0.7 R_{\odot} \leq r \leq R_{\odot}, 0 \leq \theta \leq \pi / 2\right)$ :

$$
\begin{gathered}
\frac{\partial A}{\partial t}+\frac{1}{s}\left(\mathbf{v}_{p} . \nabla\right)(s A)=\eta\left(\nabla^{2}-\frac{1}{s^{2}}\right) A+Q \\
\frac{\partial B}{\partial t}+\frac{1}{r}\left[\frac{\partial}{\partial r}\left(r v_{r} B\right)+\frac{\partial}{\partial \theta}\left(v_{\theta} B\right)\right]=\eta\left(\nabla^{2}-\frac{1}{s^{2}}\right) B+s\left(\mathbf{B}_{p} . \nabla\right) \Omega
\end{gathered}
$$


where $s=r \sin \theta$ and $\mathbf{B}_{p}=\nabla \times\left(A \mathbf{e}_{\phi}\right)$ is the poloidal field and $B$ is the toroidal field. We have added one extra term $Q$ on the right-hand side of equation (1), which governs the evolution of the poloidal field. This term does not follow from the induction equation. The usual $\alpha \Omega$ dynamo is given by the equations (1) and (2), with $Q=\alpha B$. We use the same distribution of $\alpha$ and $\Omega$ as in Choudhuri et al. (1995)-taking $\alpha$ concentrated near the surface and a layer of radial shear at the bottom of the convection zone.

We now adopt the following algorithm to incorporate buoyancy in our model:

* At intervals of time $\tau$, we check whether the toroidal field $B$ exceeds a certain critical field $B_{c}$, anywhere in the bottom of the SCZ.

* If $B$ exceeds $B_{c}$ at any point near the bottom of the SCZ, then a certain fraction $f$ of it is made to erupt to regions close to the surface, where the $\alpha$ effect is concentrated.

* The erupted field is algebraically added to the field already present near the surface and the field at the bottom from where the eruption takes place is appropriately depleted, so that flux is conserved in the whole process.

The $\alpha$ effect works on this erupted field and generates the weak and diffuse poloidal field that we see on the surface-the essence of which is captured by the phenomenological source term $Q$ in our equation (1).

There are three parameters which define this flux eruption process completely. One is $\tau$ (the time between successive eruptions) which we fix at $\tau=8.8 \times 10^{5} \mathrm{~s}$, approximately corresponding to an order of a thousand eruptions in a complete dynamo period. The second is $B_{c}$, the critical field, for which we use a value of $B_{c}=1$ (in dimensionless units), for most of the calculations. The third is the fraction $f$ of the toroidal field which is made to erupt. We study the dependence of the dynamo on some of these parameters and present our main results in the next section. The only nonlinearity in our problem comes through the $\alpha$-quenching which is adjusted such that the peak value of the magnetic field turns out to be of order unity.

\section{Results}

In Fig. 1 we plot the variation of the dynamo time period $T_{d}$ with the control parameter $f$, for three different values of the critical field $B_{c}$. It is seen that $T_{d}$ decreases with increasing $f$ and reaches an asymptotic value of 25.5 years. The fall in the value of $T_{d}$ with $f$ is quite drastic and it is obvious that making magnetic buoyancy more important (by increasing the control parameter $f$ ) has a significant effect on the working of the dynamo.

The primary role of buoyancy is to transport toroidal flux from the bottom of SCZ to the top. By making buoyancy stronger (with larger $f$ ), one makes the flux transport process more rapid and thereby reduces the dynamo period. Also, as the toroidal field belt travels equatorward with the progress of the dynamo cycle as is apparent from Fig. 2 (left), its strength decreases due to eruptions and becomes lower than $B_{c}$. In such a scenario, if $B_{c}$ is also decreased, then we will find eruptions at lower and lower latitudes, which will effectively increase the zone of activity of the dynamo. This will result in an increase in the dynamo period 


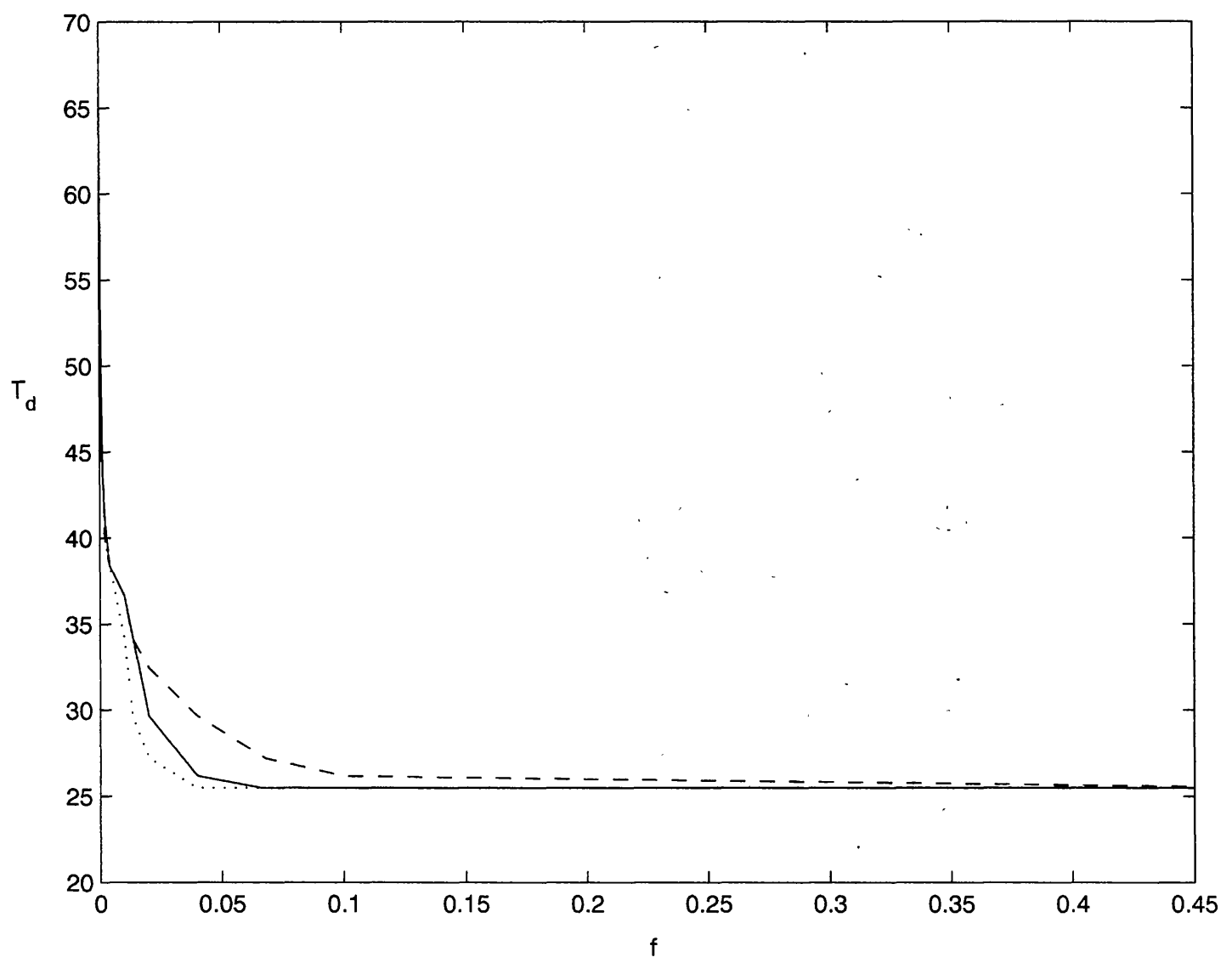

Figure 1. $T_{d}$ in years vs $f$ : the dotted line is for $B_{c}=2.0$, the solid line for $B_{c}=1.0$ and the dashed line for $B_{c}=0.5$.

and obviously it will fall more slowly with $f$ (for a lower $B_{c}$ ) as is evident from Fig. 1.

Fig. 2 (left) is a butterfly diagram for the toroidal field near the bottom of the SCZ. This figure is obtained with $B_{c}=1$ and $f=0.1$ and the contour levels that we have plotted are: $0.5,1,2,4,8$ and 16 respectively. We clearly see an equatorward propagation of the fields with time, in keeping with the equatorward propagation of sunspots. Note however that we do not see any strong toroidal field belt at low latitudes near the equator, which may be contradictory to observations of sunspots at low latitudes in the Sun. The reason for this becomes clear when we look at the plot of eruption latitude vs time, shown in Fig. 2 (right). We see that eruptions start occuring at very high latitudes-where we find the strongest toroidal fields, a feature present in Durney's (1997) results as well.

It is clear that all the recent calculations on Babcock-Leighton type solar dynamos are still of rather exploratory nature, since none of the authors have succeeded yet in producing butterfly diagrams resembling observations closely. With the basic physics of incorporating magnetic buoyancy having been explored, we are now in the process of developing more realistic models of the dynamo incorporating a solar-like profile of the angular velocity distribution and other improvements. It remains to be seen if the improved models will succeed in explaining the observational data more satisfactorily. 


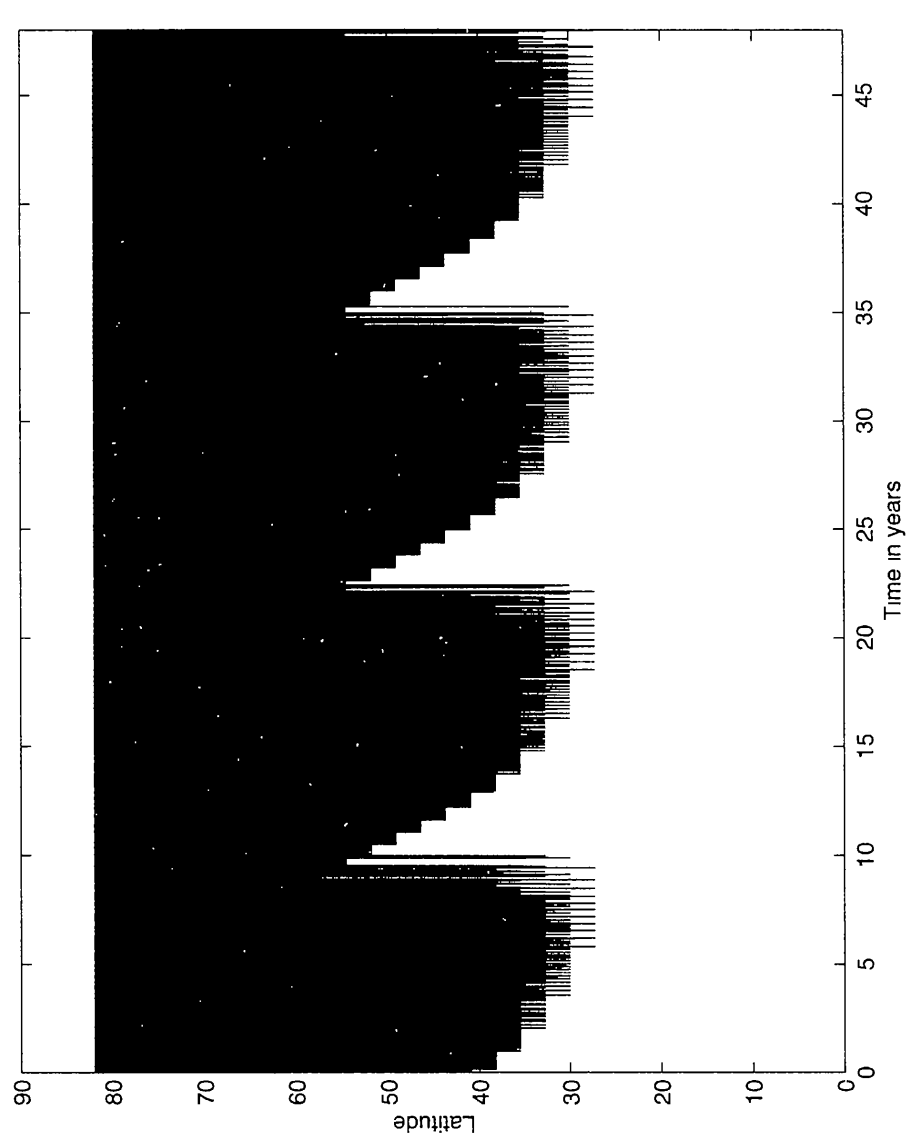

$\infty$

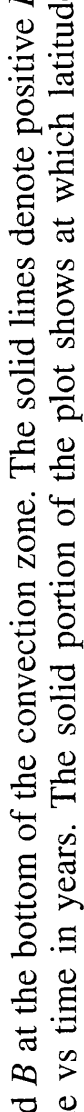

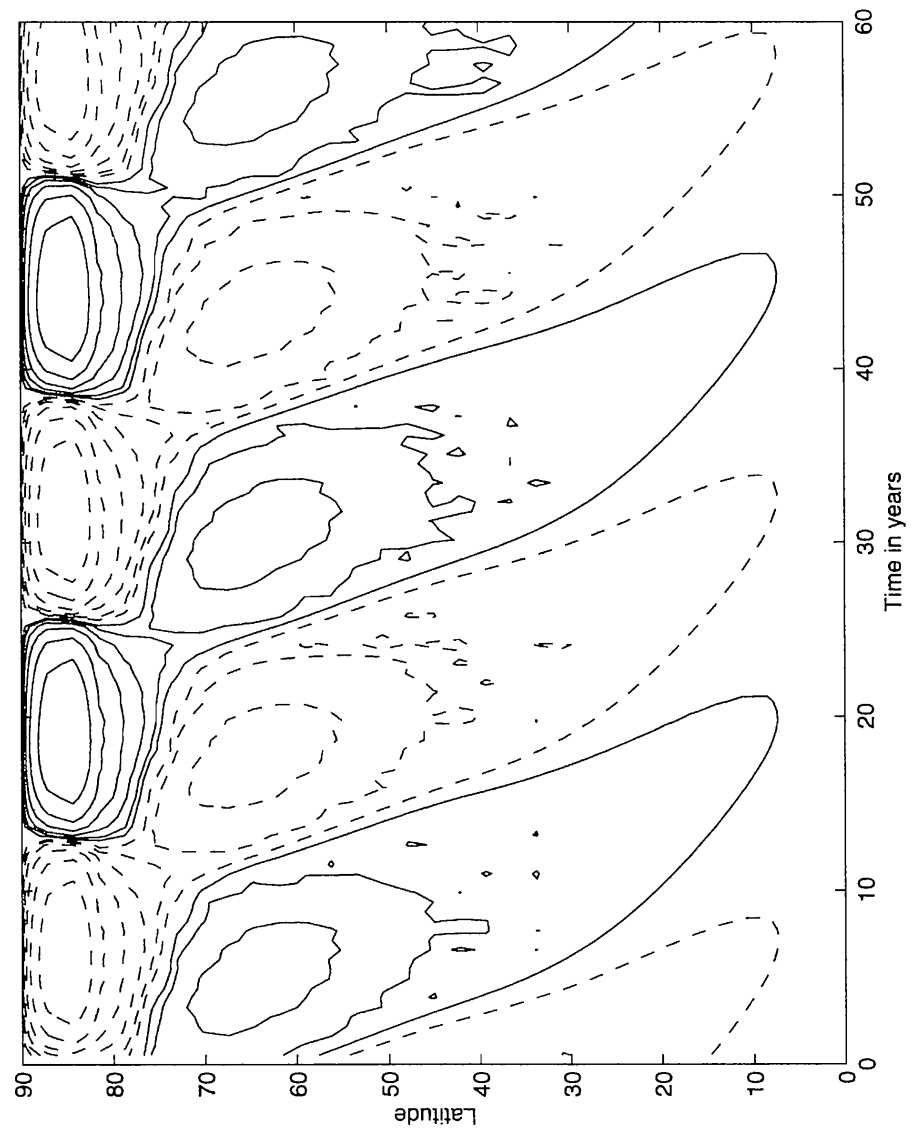

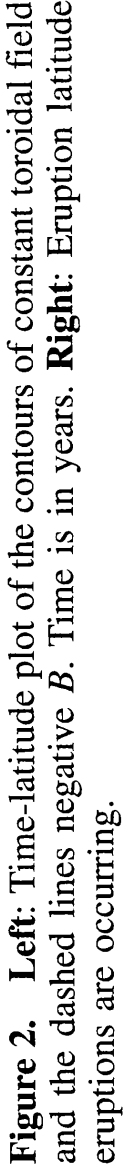




\section{References}

Choudhuri, A. R. 2000, these proceedings.

Choudhuri, A. R., Schüssler M., Dikpati M. 1995, Astr. Astrophys., 303, L29.

Dikpati, M., Charbonneau, P. 1999, Astrophys. J., 518, 508.

Durney, B. R. 1997, Astrophys. J., 486, 1065. 